\title{
Measurement of the Density Profile of a Toroidal Non-neutral Plasma with a Wall-Probe Array
}

\author{
Haruhiko SAITOH, Yoshihisa YANO, Tatsunori MIZUSHIMA, Junji MORIKAWA \\ and Zensho YOSHIDA \\ Department of Advanced Energy, Graduate School of Frontier Sciences, The University of Tokyo, \\ 5-1-5 Kashiwanoha, Kashiwa, Chiba 277-8561, Japan
}

(Received 30 August 2009 / Accepted 3 October 2009)

\begin{abstract}
The Ring Trap 1 (RT-1) device can confine a pure-electron plasma in the magnetospheric dipole field configuration [Z. Yoshida et al., Plasma Fusion Res. 1, 008 (2006); Y. Ogawa et al., Plasma Fusion Res. 4, 020 (2009); H. Saitoh et al., Plasma Fusion Res. 2, 045 (2007)]. While this system has proved long-term (typically about $400 \mathrm{~s}$ ) stable confinement, the internal structure of the electron cloud has not been well understood. The spatial distribution of the charge density has been estimated using an array of wall probes. Multiple wall probes with current amplifiers and analog integration circuits were developed and used to estimate image charge profiles on the chamber walls. The electrons are trapped inside the separatrix during the injection phase. In the stable confinement phase, electrons shift toward the stronger magnetic field region and produce a rather peaked profile.
\end{abstract}

(C) 2009 The Japan Society of Plasma Science and Nuclear Fusion Research

Keywords: non-neutral plasma, internal coil device, magnetospheric configuration

DOI: $10.1585 /$ pfr.4.054

\section{Introduction}

The Ring Trap 1 (RT-1) device is a "laboratory magnetosphere" that produces a magnetospheric dipole field configuration by a levitated superconducting coil [1,2]. A pure electron plasma can be confined in RT-1; the density is of the order of $10^{11} \mathrm{~m}^{-3}$, and the confinement time is longer than $400 \mathrm{~s}$ [3]. A toroidal system [4-14] has merits in trapping various kinds of charged particles including antimatters [15-18].

In our previous experiment on the Proto-RT device [6-8], we measured the internal electric potential by inserting probes and showed that an appropriate control of the potential of the internal ring produces closed equi-potential surfaces aligned with the magnetic surfaces, achieving good confinement. However, the profile of the density or potential distribution is not known in the long-confinement state, because probing destroys the long confinement. In linear configurations such as a Penning-Malmberg trap [19], the confined charged particles can be dumped toward the device ends along the field lines by controlling the trapping electrostatic potential barriers. Good reproducibility of the plasma and the use of sensitive imaging sensors or energy analyzers made possible precise measurements of the confinement properties and distribution reconstruction of linear non-neutral plasmas [20]. However, in toroidal geometries, where charged particles are trapped inside closed field lines, we cannot apply such a method of measuring internal structures. Azimuthal symmetry of the system is essential to conserve the canonical angular momentum of particles and to realize good confinement [6]. Langmuir probes are useful tools for measuring local plasma parameters $[7-9,11]$ during electron injection, but they cause serious disturbance and cannot be used in the stable confinement phase of plasmas, especially those consisting of small numbers of particles such as antimatters. Perturbation-free diagnostics of density profile information is, therefore, strongly required for experiments on toroidal non-neutral plasmas.

In this study, multiple wall probes [4] were used for perturbation-free diagnostics of radial electric field strength and spatial profiles of the plasma, as well as electrostatic fluctuation measurements in RT-1. To minimize electrostatic perturbations, image currents were measured using current amplifiers, where there were no voltage differences between the walls and the chamber. The image current signals were integrated by analog amplifiers to measure local electric field strengths, which were proportional to the image charge densities on the walls. Using three walls located inside the chamber and a simplified assumption of plasma shapes, the spatial profiles of the electron plasma were estimated. During electron injection, the plasma was located in the whole region inside the separatrix. After the electron supply is terminated, the plasma moves inward, i.e., toward the stronger field region. The experimental apparatus and procedure for electric field measurements by the wall probes are described in Sec. 2. Experimental results are reported in Sec. 3, and estimated electron distributions in RT-1 are reported in Sec. 4. 


\section{Experimental Setup and Diagnos- tics}

Figure 1 shows the poloidal cross section of RT-1 $[1,2]$. Inside the chamber, RT-1 has a high-temperature superconducting coil with a Bi-2223 wire rod. The coil was levitated by using a feedback-controlled levitation electromagnet to minimize disturbances to the plasma. The application of the high-temperature superconducting coil has made possible six hours of plasma experiments with coil levitation before the limitation set by the coil temperature increase. A more detailed description of the RT-1 device and the superconducting levitated coil system can be found in Ref. [2]. The vacuum chamber had an inner diameter of $2 \mathrm{~m}$ and was evacuated to a base pressure of $7 \times 10^{-7} \mathrm{~Pa}$ by two turbomolecular pumps. The magnetic field was generated by a combination of the levitated superconducting magnet and the levitation coil. Each of the coils was operated with a current of $250 \mathrm{kAT}$ and $28.8 \mathrm{kAT}$, respectively. For geomagnetic field compensation, another set of coils was located outside the chamber [21]. As illustrated in Fig. 1, the typical magnetic field strength in the confinement region varied from $B=0.01$ to $0.1 \mathrm{~T}$. On the mid-plane of the device, the separatrix of the magnetic surfaces (shown as bold lines in the figure) was located at $r=92 \mathrm{~cm}$.

Electrons were injected by an electron gun with a $\mathrm{LaB}_{6}$ cathode located at the edge of the confinement re-

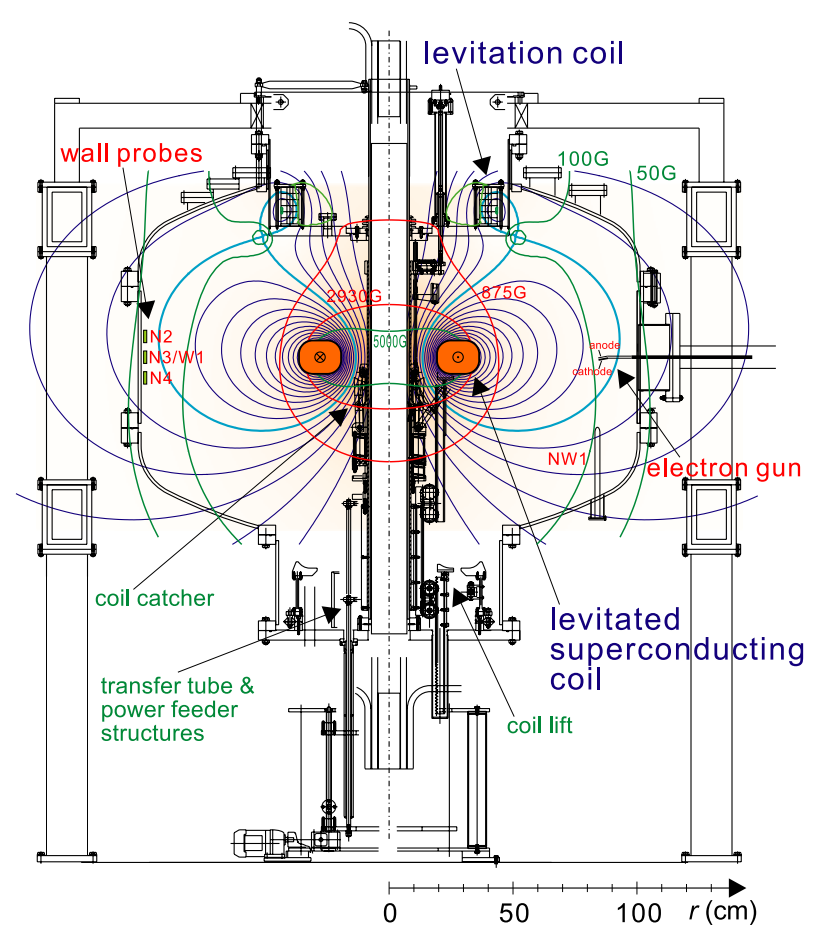

Fig. 1 Cross-sectional view of RT-1 including coil magnets, vacuum chamber, and diagnostic probes. Thin lines show magnetic surfaces generated by the combination of levitated superconducting coil and normal-conducting levitation coil. gion [7]. The cathode was heated by a DC current of $24 \mathrm{~A}$, and it was negatively biased to $-V_{\text {acc }}$ against the vacuum chamber. Thermionic electrons from the cathode were accelerated between the cathode and a mesh anode that was electrically connected to the chamber wall. As shown in Fig. 1, the angle between the electron beam and $+\hat{z}$ was $11^{\circ}$, when the gun was set to inject electrons in the upward direction. The electron gun can be rotated about the axis, and in the present experiment, the electron injection direction was set as $v_{r}: v_{\theta}: v_{z}=-0.194: 0: 1$. The cathode current, anode current, and beam current were monitored using $1 \Omega$ resistances. The electron gun axis was located on the equatorial plane of the chamber $(z=0 \mathrm{~cm})$ at the southeast port of RT-1, and was movable in the radial direction from $r=65$ to $120 \mathrm{~cm}$. In the present experiment, the electron gun cathode was located at $r_{\text {gun }}=75 \mathrm{~cm}$ and $V_{\text {acc }}=150 \mathrm{~V}$.

The electrostatic fluctuations and radial electric field strengths of the plasma were measured by the wall probes. The walls were stainless-steel foils, installed from the north ports of RT- 1 and located at $r=1 \mathrm{~m}$, as illustrated in Fig. 1. The walls were located outside the confinement region, and positive image charges $Q_{\mathrm{i}}$ were induced on them as the plasma was generated. Walls N2-N4 were circular with a diameter of $5 \mathrm{~cm}$ and were used to measure the radial electric field strength $E_{\mathrm{r}}$. The vertical positions of each probe were N2: $z=+12 \mathrm{~cm}, \mathrm{~N} 3: z=0 \mathrm{~cm}$, and N4: $z=-12 \mathrm{~cm}$. Another wall (W1) with a diameter of $13 \mathrm{~cm}$ was located at $z=0 \mathrm{~cm}$ at the west port of RT-1.

In order to minimize plasma disturbances, the image currents were measured by analog current amplifiers; a circuit diagram is shown in Fig. 2. There were no voltage differences between the wall probes and the chamber
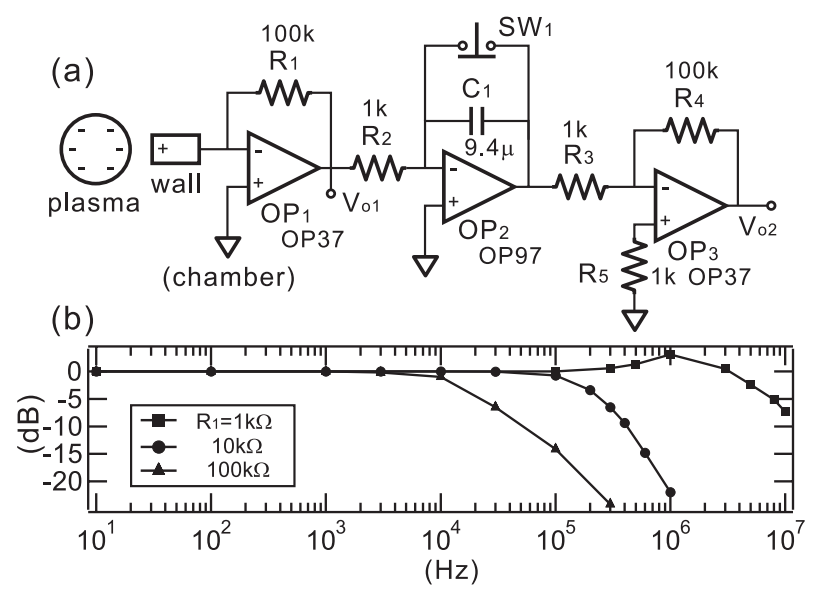

Fig. 2 (a) Amplifier circuit diagram for wall probe used for electrostatic fluctuations and $E_{\mathrm{r}}$ measurements. Circuit consists of current amplifier, integrator, and inverting amplifier which also works as a buffer between the amplifier and $\mathrm{AD}$ converter. $\mathrm{SW}_{1}$ is a gate switch for the integrator. (b) Normalized frequency characteristics of current amplifier with different $R_{1}$. 
due to the virtual short circuit between the inverting and non-inverting inputs of $\mathrm{OP}_{1}$, which contributed to the axisymmetry of the trap system. The amplified signals of the image current $I_{\mathrm{i}}=\mathrm{d} Q_{\mathrm{i}} / \mathrm{d} t, V_{\mathrm{o} 1}$, were sent to analog integrators to measure image charges $Q_{\mathrm{i}}$ on the walls so that we could simultaneously measure the electrostatic fluctuations and $E_{\mathrm{r}}$ of the plasma. The values of the resistors and a capacitor in the circuit were chosen to provide a good time response for the fluctuation measurements or to ensure good signal-to-noise ratios of the integrated output. The values used in the present experiment are shown in Fig. 2 (a). Since the image charge density induced on a wall facing a plasma is

$$
\sigma_{\mathrm{i}}=-\varepsilon_{0} E_{\mathrm{r}}
$$

and the image charge is calculated from

$$
V_{\mathrm{o} 2}=-\frac{R_{1} R_{5}}{C_{1} R_{2} R_{3}} \int I_{\mathrm{i}} \mathrm{d} t=-1.06 \times 10^{10} Q_{\mathrm{i}},
$$

the averaged electric field strength at the position of the wall is evaluated as

$$
E_{\mathrm{r}}=-\int I_{\mathrm{i}} \mathrm{d} t / \varepsilon_{0} S=5.33 \times 10^{3} \times V_{\mathrm{o} 2},
$$

where $S=1.96 \times 10^{-3} \mathrm{~m}^{2}$ is the wall area facing the plasma, $\varepsilon_{0}$ is the electric permittivity of vacuum, and $V_{\mathrm{o} 2}$ is the output of the amplifier illustrated in Fig. 2 (a). The longest applicative integration time of the circuit was approximately $10 \mathrm{~s}$ because of the drift of the integrated signals caused by the offset current and offset voltage of the amplifier. Variations of $E_{\mathrm{r}}$ with a time scale longer than $10 \mathrm{~s}$ were out of the measurable range in the present experiment.

\section{Experimental Results}

In RT-1, stabilization of electrostatic fluctuations and long-time confinement of toroidal non-neutral plasma were realized by coil levitation [3]. Figure 3 shows the typical

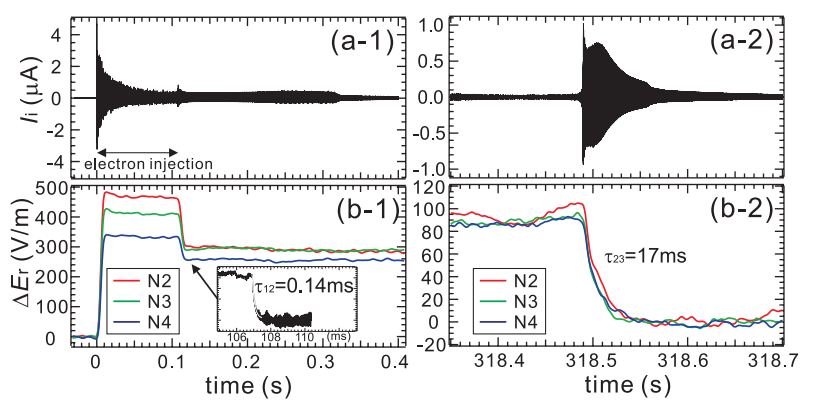

Fig. 3 (a) Electrostatic fluctuations measured by wall W1 and (b) radial electric field strength measured by walls $\mathrm{N} 2$, $\mathrm{N} 3$, and N4, when (1) electrons were injected and (2) confinement ended. Numerically integrated fluctuation data are also displayed in (b-1). waveforms of (a) electrostatic fluctuations and (b) $E_{\mathrm{r}}$ measured by the wall probes. Because the electron confinement time was much longer than the measurable time of the integration circuit, the integrated signals might contain some offsets of $E_{\mathrm{r}}$. We could only estimate the variation of $E_{\mathrm{r}}, \Delta E_{\mathrm{r}}$. Figure $3(\mathrm{~b})$ plots $\Delta E_{\mathrm{r}}$ as variations from $E_{\mathrm{r}}(1)$ before electron injection and (2) after confinement ended. In Fig. 3 (1), electrons were injected from $t=0$ to $0.107 \mathrm{~s}$ by the electron gun. The cathode heating current as well as $V_{\text {acc }}$ were turned off after $t=0.107 \mathrm{~s}$, in order to ensure that the electron supply was terminated. During the electron injection, the initially observed large amplitude decayed approximately exponentially, and the averaged $\Delta E_{\mathrm{r}}$ at this period were $\mathrm{N} 2: 456 \mathrm{Vm}^{-1}, \mathrm{~N} 3: 412 \mathrm{Vm}^{-1}$, and N4: $334 \mathrm{Vm}^{-1}$. No substantial variation of $\Delta E_{\mathrm{r}}$ occurred from $t=0$ to $0.107 \mathrm{~s}$, indicating that the plasma was in a steady state where the injected beam current $I_{\mathrm{b}}$ balanced the loss current. The confinement time $\tau=Q / I_{\mathrm{b}}$ in this period, where $Q$ is the total electron charge, was estimated to be of the order of $10^{-3} \mathrm{~s}$, which was five orders of magnitude shorter than the stable confinement time.

A rapid decrease of $\Delta E_{\mathrm{r}}$ was observed after the termination of the electron supply at $t=0.107 \mathrm{~s}$, but approximately $70 \%$ of the initial $\Delta E_{\mathrm{r}}$ remained to be observed, as shown in Fig. 3 (b-1). The decay time of $\Delta E_{\mathrm{r}}$ at this point was faster than the integration circuit time constant, and according to the numerical integration of the fluctuation signal, $\tau_{12}=0.14 \mathrm{~ms}$. The loss mechanism of electrons at this point will be described in the next section. $\Delta E_{\mathrm{r}}$ just after the electron supply ended was $\mathrm{N} 2: 296 \mathrm{Vm}^{-1}, \mathrm{~N} 3$ : $296 \mathrm{Vm}^{-1}$, and N4: $257 \mathrm{Vm}^{-1}$. The fluctuation attenuated further at $t=0.32 \mathrm{~s}$, and quiescent confinement was realized subsequently. As shown in Fig. 3 (2), the confinement ended at $t=318.48 \mathrm{~s}$ with an onset of instability and decrease in $\Delta E_{\mathrm{r}} . \Delta E_{\mathrm{r}}$ at $t=318.4 \mathrm{~s}$ was $\mathrm{N} 2: 89.7 \mathrm{Vm}^{-1}$, N3: $89.3 \mathrm{Vm}^{-1}$, and $\mathrm{N} 4: 86.2 \mathrm{Vm}^{-1}$, and the differences between each wall signal were quite small when compared with those during the electron injection. At the end of confinement, the decay time constant of $\Delta E_{\mathrm{r}}$ was $\tau_{23}=17 \mathrm{~ms}$, which was an order of magnitude longer than $\tau_{12}$.

When $V_{\text {acc }}$ was optimized and stable confinement was realized, no substantial change was observed in the fluctuation frequencies at the end of the electron injection, while a clear drop of $E_{\mathrm{r}}$ was observed. Figure 4 plots the fundamental frequencies of the fluctuation and $\Delta E_{\mathrm{r}}$ during electron injection (circles) and stable confinement (triangles). The data points are divided into two groups with different dependencies of the frequency on $\Delta E_{\mathrm{r}}$, implying that the plasma had different spatial distributions. Also, the extrapolated data apparently do not intersect the origin of the graph, indicating that a steady component of $E_{\mathrm{r}}$ was not detected by the present integrator circuit. In RT-1, the levitated superconducting coil was covered by a stainlesssteel case that was charged up to certain voltages $V_{\mathrm{c}}$ by the formation of an electron plasma, though direct measurements of $V_{\mathrm{c}}$ were not carried out. In previous experiments 


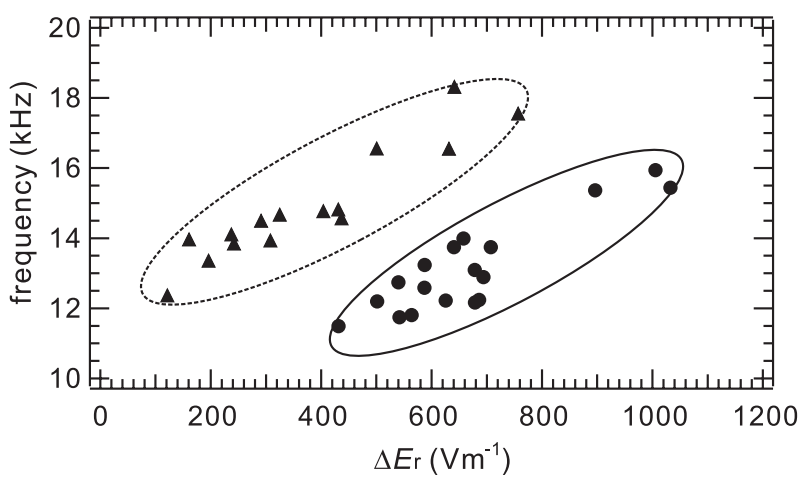

Fig. 4 Fundamental frequencies of fluctuation and $\Delta E_{\mathrm{r}}$ measured by wall probes during electron injection (circles) and stable confinement phase (triangles).

in Proto-RT [8] with a mechanically supported dipole field coil, we found $V_{\mathrm{c}}$ to be typically $20 \%$ lower than $-V_{\text {acc }}$ according to the measurements by a $100 \mathrm{M} \Omega$ high impedance voltage probe. Because the dipole field coil in RT-1 was levitated and electrically insulated from the chamber, it is quite probable that the coil was charged up in the same manner as in Proto-RT.

\section{Estimation of Plasma Profiles}

In this section, the spatial profiles of the plasma are estimated using the signals of $E_{\mathrm{r}}$ measured by walls N2N4. We made the following assumptions for the estimation. According to the explanation in the previous section, we assumed that the levitated coil case was charged up to $V_{\mathrm{c}}=-1.2 \times V_{\mathrm{acc}}$ and it was temporally constant, because electrons were repeatedly injected with the same $V_{\text {acc }}=150 \mathrm{~V}$ in the present experiments. We also assumed that the plasma has a simplified shape, as described in the following sections and in Fig. 5(a). Using these assumptions, we numerically solved the Poisson equation and looked for plasma profiles that were consistent with the observation of the wall probes. In the numerical calculation of electrostatic potential profiles, we use the substitute charge method satisfying the boundary conditions on the chamber and the levitated coil [22] of RT-1. Again, we note that the wall probes cannot measure the steady component of $E_{\mathrm{r}}$ generated by the levitated coil with $V_{\mathrm{C}}$.

We write the total charge of the system as

$$
\rho(\boldsymbol{r})=\rho_{\mathrm{i} 0}(\boldsymbol{r})+\rho_{\mathrm{s}}(\boldsymbol{r})+\rho_{\mathrm{i}}(\boldsymbol{r}),
$$

where $\rho_{\mathrm{i} 0}(\boldsymbol{r})$ represents the image charges induced by the levitated coil with $V_{\mathrm{c}}$ in a vacuum without space charges, $\rho_{\mathrm{s}}(\boldsymbol{r})$ is the space charges of electrons, and $\rho_{\mathrm{i}}(\boldsymbol{r})$ is the image charges induced by $\rho_{\mathrm{s}}(\boldsymbol{r})$ satisfying the boundary condition $V_{\mathrm{c}}=0 \mathrm{~V}$ (the levitated coil is electrically connected to the chamber). The above-mentioned $\Delta E_{\mathrm{r}}$ values that can be measured by the wall probes were generated by $\rho_{\mathrm{s}}(\boldsymbol{r})+\rho_{\mathrm{i}}(\boldsymbol{r})$, because $\rho_{\mathrm{i} 0}(\boldsymbol{r})$ generated the steady component

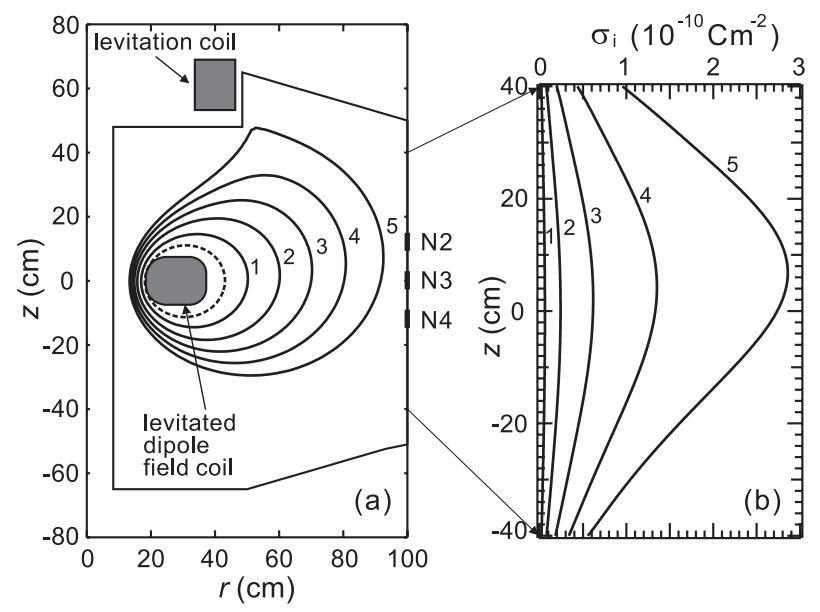

Fig. 5 (a) Poloidal cross section of RT-1 and confinement regions of plasma and (b) image charge density profiles on vacuum chamber at $r=1 \mathrm{~m}$ with various outer plasma edges 1-5 (solid lines) and fixed inner edge (dotted line). Outer edge surfaces were located at 1: $r_{0}=50 \mathrm{~cm}, 2$ : $60 \mathrm{~cm}, 3: 70 \mathrm{~cm}, 4: 80 \mathrm{~cm}$, and $5: 50 \mathrm{~cm}$ at $z=0 \mathrm{~cm}$.

of $E_{\mathrm{r}}$. For $\rho_{\mathrm{s}}(\boldsymbol{r})$ and $V_{\mathrm{c}}$, we calculated potential profiles by the Poisson equation,

$$
\begin{aligned}
\phi(\boldsymbol{r}) & =\frac{1}{4 \pi \varepsilon_{0}} \int \frac{\rho\left(\boldsymbol{r}^{\prime}\right) \mathrm{d} \boldsymbol{r}^{\prime}}{\left|\boldsymbol{r}^{\prime}-\boldsymbol{r}\right|} \\
& =\frac{1}{\pi \varepsilon_{0}} \iint \rho\left(r^{\prime}, z^{\prime}\right) \frac{k}{2} \sqrt{\frac{r^{\prime}}{r}} K(k) \mathrm{d} r^{\prime} \mathrm{d} z^{\prime}
\end{aligned}
$$

satisfying the boundary conditions. Here axisymmetry $\partial / \partial \theta=0$ of the system was assumed, and $K(k)$ is the elliptic integral of the first kind where $k^{2}=4 r r^{\prime} /\left[\left(r+r^{\prime}\right)^{2}+\right.$ $\left.\left(z-z^{\prime}\right)^{2}\right]$.

We assumed simplified plasma shapes with uniform densities, because the number of the wall probes was small and precise reconstruction of the density distribution was out of the range of the present study. In RT-1, electrons were injected with an initial velocity of $v_{\mathrm{e}}=\sqrt{2 e V_{\mathrm{acc}} / m_{\mathrm{e}}}$, and the electrons filled the magnetic surfaces where the electron gun was located by a combination of thermal motion and toroidal motion with the $\nabla B$ and curvature drift velocity. The electrons on the thin surfaces were then radially transported across the magnetic surfaces to fill the rest of the confinement region until equilibrium density profiles were built up. Once the self-radial electric fields were generated, electrons were transported in the toroidal direction by the strong $\boldsymbol{E} \times \boldsymbol{B}$ drift motion. The toroidal rotation time of electrons with $v_{\boldsymbol{E} \times \boldsymbol{B}} \approx 5 \times 10^{4} \mathrm{~ms}^{-1}$, of the order of $10^{-4} \mathrm{~s}$, was much shorter than the observed confinement time of the plasma, and the plasma parameters can be functions of magnetic surface even in a poloidal magnetic field. Because $\omega_{\mathrm{p}}{ }^{2} / \omega_{\mathrm{c}}{ }^{2} \approx 0.01 \ll 1$ was satisfied and the plasma current was negligibly small, we need to consider only the magnetic field generated by the external coils. Here, $\omega_{\mathrm{p}}$ is the plasma angular frequency and $\omega_{\mathrm{c}}$ is the electron cyclotron angular frequency. We therefore assumed that the 


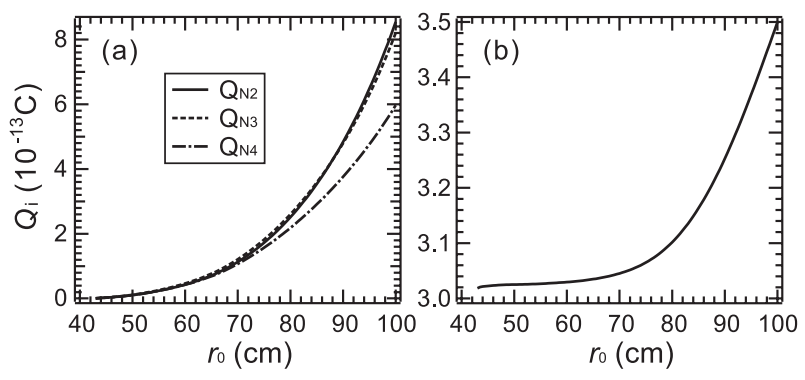

Fig. 6 (a) Image charges $Q_{\mathrm{N} 2}, Q_{\mathrm{N} 3}$, and $Q_{\mathrm{N} 4}$ induced on each wall probe in variation of plasma shapes. (b) Calculated $\left(Q_{\mathrm{N} 2} / Q_{\mathrm{N} 4}\right)^{2}+\left(Q_{\mathrm{N} 3} / Q_{\mathrm{N} 4}\right)^{2}$, used to indicate variation of each wall signal.

plasma was axisymmetric and was confined inside the edge surfaces that coincided with the vacuum magnetic surfaces. The outermost magnetic surface that intersected the dipole field coil, illustrated as a dotted line in Fig. 5 (a), was chosen as the inner edge of the plasma. This is because the coil surface was a major loss channel of electrons, and we assumed that the plasma was not confined inside this region. The inner edge surface was located at $r_{0}=43 \mathrm{~cm}$ at $z=0 \mathrm{~cm}$. Figure 5 (b) shows image charge profiles on the chamber wall at $r=100 \mathrm{~cm}$ at various outer edge surfaces of the plasma with a fixed inner edge surface. As the outer edge was moved outward and the plasma was shifted close to the chamber wall, $\sigma_{\mathrm{i}}$ showed peaked profiles and was observed as an increase in variation between $\Delta E_{\mathrm{r}}$ signals on walls N2-N4.

In Fig. 6(a), image charges induced on walls N2-N4, $Q_{\mathrm{N} 2}-Q_{\mathrm{N} 4}$, were calculated at various locations $r_{0}$ of the outer edge surfaces of the plasma. $\sigma_{\mathrm{i}}$ was simply integrated on the wall area to obtain the induced charges on the walls, because each wall was electrically shorted to the chamber, and distortion of $E_{\mathrm{r}}$ due to the wall potentials could be neglected. Because of the asymmetry of the magnetic surfaces generated by the combination of the levitated and levitation coils, walls N2 and N3 rather than N4 were close to the plasma, and relatively small signals were observed on N4. To evaluate the relationship between the plasma shapes and the observed $E_{\mathrm{r}}$ on N2-N3, $\left(Q_{\mathrm{N} 2} / Q_{\mathrm{N} 4}\right)^{2}+\left(Q_{\mathrm{N} 3} / Q_{\mathrm{N} 4}\right)^{2}$ were calculated with variouos values of $r_{0}$. As shown in Fig. $6(\mathrm{~b}), r_{0}$ can be uniquely determined from this value, and we can use it to estimate the plasma profiles.

Using the method described above, the outer edge surfaces of the plasma were estimated in Fig. 7 for the typical cases of (1) during electron injection, (2) just after the electron supply ended, and (3) just before the end of confinement for the data shown in Fig. 3. The radial positions of the plasma outer edge $r_{0}$ at $z=0 \mathrm{~cm}$ were calculated as (1) $91.6 \mathrm{~cm}$, (2) $77.9 \mathrm{~cm}$, and (3) $60.5 \mathrm{~cm}$. The confinement region was observed to gradually shift toward the stronger magnetic field region. Figure 7 (a) shows the
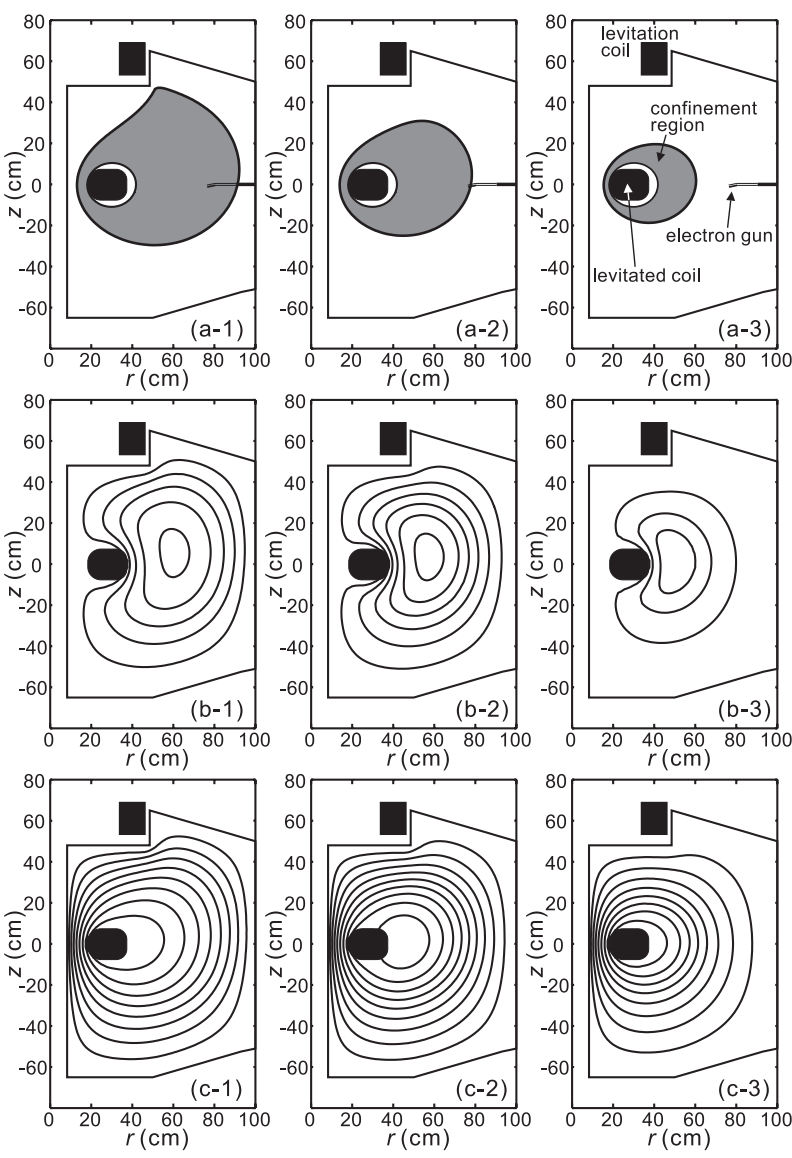

Fig. 7 (a) Confinement regions of plasma estimated by wall probes, (b) electrostatic potential profiles generated by $\rho_{\mathrm{s}}+\rho_{\mathrm{i}}$ that can be measured by wall probes, and (c) total potential profiles generated by $\rho_{\mathrm{i} 0}+\rho_{\mathrm{s}}+\rho_{\mathrm{i}}$ where $V_{\mathrm{c}}=-1.2 \times V_{\text {acc }}=-180 \mathrm{~V}$ was assumed, (1) during electron injection, (2) just after electron injection ended, and (3) just before confinement ended. Contours of potential profiles are plotted with intervals of $20 \mathrm{~V}$.

electron density distributions in the poloidal cross section of RT-1 for the three cases. The uniform electron number densities that best reproduced the observed $E_{\mathrm{r}}$ on the walls were (1) $1.4 \times 10^{11} \mathrm{~m}^{-3}$, (2) $2.4 \times 10^{11} \mathrm{~m}^{-3}$, and (3) $3.2 \times 10^{11} \mathrm{~m}^{-3}$, and the trapped electron numbers were (1) $1.9 \times 10^{11}$, (2) $1.7 \times 10^{11}$, and (3) $8.0 \times 10^{10} . E_{\mathrm{r}}$ generated by the density profiles in Fig. 7 (a) with these densities and those of the measured values are shown in Fig. 8. Fairly good agreement was found between the two values, indicating that the simplified assumption of the plasma shapes was valid as a rough approximation of the plasma distributions. In the present analysis, density variations in the confinement region, which can result from the strongly inhomogeneous dipole field or other causes, were not evaluated. The remaining small disagreement between the measured and calculated $E_{\mathrm{r}}$ should be attributed to these effects. Consideration of a more detailed reconstruction of the density profiles in future experiments is described in the next section. 


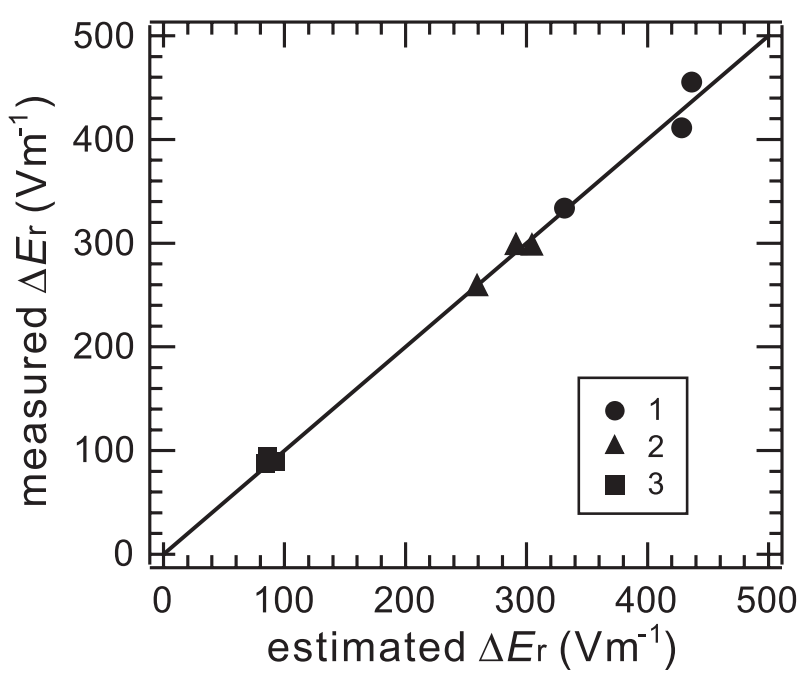

Fig. 8 Values of $\Delta E_{\mathrm{r}}$ measured by wall probes N2-N4 and estimated $\Delta E_{\mathrm{r}}$ generated by electron clouds in Fig. 7 (a), (1) during electron injection, (2) just after electron injection ended, and (3) just before confinement ended.

During the electron injection, the outer edge of the electron plasma closely agreed with the separatrix located at $r_{0}=92 \mathrm{~cm}$, as shown in Fig. 7 (a-1). The Larmor radius of an electron with $T_{\mathrm{e}}=150 \mathrm{eV}$ was $6.0 \mathrm{~mm}$ at the separatrix on the $z=0 \mathrm{~cm}$ plane, and electrons were considered to be trapped approximately inside the separatrix. Thus, the estimated plasma density profile was consistent with the expected electron distribution. When the electron supply ended at $t=0.107 \mathrm{~s}$, electrons located on the magnetic surfaces between the separatrix and the electron gun at $r_{\text {gun }}=75 \mathrm{~cm}$ were selectively lost (Fig. 7 (a-2)). In this region, the electron gun body and its supporting structure intersected the magnetic surfaces, and it was a loss channel of electrons. The decay time of electrons due to collisions with the electron gun was comparable to the observed decrease of $E_{\mathrm{r}}$ described in the previous section. The motion of the guiding center of electrons consisted of fast longitudinal motion with $v_{\mathrm{e}} \approx 6 \times 10^{5} \mathrm{~ms}^{-1}$ on the $z=0 \mathrm{~cm}$ plane and slower toroidal drift motion with $v_{\boldsymbol{E} \times \boldsymbol{B}}=4 \times 10^{4} \mathrm{~ms}^{-1}$. By using $2 \pi r=5.2 \mathrm{~m}$ as the free path of the injected electrons in the toroidal direction, where $r=83.5 \mathrm{~cm}$ was the midpoint between the electron gun and the separatrix, the maximum confinement time of electrons before they hit the gun structure and were lost was calculated as $\tau=2 \pi r / v_{\boldsymbol{E} \times \boldsymbol{B}}=0.13 \mathrm{~ms}$. This value approximately agreed with the observed $E_{\mathrm{r}}$ decay time of $\tau_{12}=0.14 \mathrm{~ms}$ just after the electron injection ended (Fig. 3 (b-1)), indicating that the electron gun was the primary loss channel of electrons in this region. Longer confinement was observed on the magnetic surfaces located inside the electron gun, and the confinement region gradually shifted inward, as can be seen in Fig. 7 (a-3). It is well known that any perturbation with a time scale comparable to or shorter than the toroidal drift period can cause violations of the third adiabatic invariant and radial diffusion of particles in a dipole field [23]. The observed temporal evolutions of the plasma shapes and increase in electron densities were probably due to the effective inward transport of electrons into the stronger field region caused by this mechanism.

Electrostatic potential profiles generated by $\rho_{\mathrm{s}}(\boldsymbol{r})+$ $\rho_{\mathrm{i}}(\boldsymbol{r})$ that can be measured by the wall probes are shown in Fig. 7 (b). Figure 7 (c) is the estimated total potential profiles $\phi(\boldsymbol{r})$ generated by the total charge $\rho_{\mathrm{i} 0}(\boldsymbol{r})+\rho_{\mathrm{s}}(\boldsymbol{r})+\rho_{\mathrm{i}}(\boldsymbol{r})$. As described in the previous sections, we assumed that the coil case was charged up to $V_{\mathrm{c}}=-1.2 \times V_{\text {acc }}=-180 \mathrm{~V}$ and set the boundary condition. Concentric potential contours were formed around the levitated coil, and the variations of $\phi$ on magnetic surfaces were relatively small especially in the stronger magnetic field region. During and just after the electron injection (Figs. 7 (c-1) and (c-2)), the potential profiles vary significantly on the magnetic surfaces. In contrast, at the final stage of the confinement (Fig. 7 (c$3)$ ), the potential contours were quite close to the magnetic surfaces, especially in the confinement region, indicating that the potential profiles were approximately functions of the magnetic surfaces.

\section{Conclusion}

Direct measurements of $E_{\mathrm{r}}$ of a toroidal non-neutral plasma have been carried out on RT-1, using a wall-probe array. We used three wall probes and estimated the confinement regions of the plasma for the typical cases of (1) during electron injection, (2) just after the electron supply ended, and (3) just before the confinement ended. During the electron gun operation, the outer edge of the electron confinement region approximately agreed with the magnetic separatrix. After the electron supply ended, electrons on the magnetic surfaces that intersected the electron gun structures were rapidly lost. In the stable confinement period, electrons shifted inward and moved to the strong magnetic field region. Estimation of the internal structures of the plasma was beyond the scope of the present study.

This problem may be solved by increasing the number of walls and choosing their proper positions in future experiments. When the plasma volume is divided into $m$ regions and the electron density $n_{i}(i=1-m)$ can be assumed to be constant in each region, the image charge $Q_{\mathrm{i}}$ induced on each wall is given by

$$
\left[Q_{\mathrm{i}}\right]_{m \times 1}=\left[a_{i j}\right]_{m \times m}\left[n_{i}\right]_{m \times 1},
$$

where $a_{i j}$ is a proportional constant of the image charge on a wall $i$ induced by the plasma charges in region $V_{j}$. $\boldsymbol{A}=\left[a_{i j}\right]$ is chosen so that the Poisson equation satisfies the boundary condition on the chamber wall. The electron density in each region is then given by

$$
\left[n_{i}\right]_{m \times 1}=\boldsymbol{A}^{-1}\left[Q_{\mathrm{i}}\right]_{m \times 1},
$$

and detailed density profiles can be estimated by using a sufficient number of the walls. Detailed density profile 
measurement with an increased number of walls will be addressed in the future experiments in RT-1.

In the present experiment, we used only three walls; thus, the spatial resolution of the measurements was just enough to estimate the average confinement region (inside the confinement region, we assume a homogeneous electron density). Note that the wall probes were located at the chamber wall at $r=1 \mathrm{~m}$, so they are not sensitive to the charges near the superconducting coil.

\section{Acknowledgment}

The authors are grateful to Associate Professor Masaru Furukawa for his valuable comments and discussions. This work was supported by Grant-in-Aid for Scientific Research 19340170 from the Japan Society for the Promotion of Science.

[1] Z. Yoshida, Y. Ogawa, J. Morikawa et al., Plasma Fusion Res. 1, 008 (2006).

[2] Y. Ogawa, Z. Yoshida, J. Morikawa et al., Plasma Fusion Res. 4, 020 (2009).

[3] H. Saitoh, Z. Yoshida, J. Morikawa, S. Watanabe, Y. Yano and J. Suzuki, Plasma Fusion Res. 2, 045 (2007).

[4] J.D. Daugherty, J.E. Eninger and G.S. Janes, Phys. Fluids 12, 2677 (1969).

[5] P. Zaveri, P.I. John, K. Avinash and P.K. Kaw, Phys. Rev. Lett. 68, 3295 (1992).

[6] Z. Yoshida, Y. Ogawa, J. Morikawa et al., in Nonneutral Plasma Physics III, AIP Conf. Proc. No. 498 (AIP, New York, 1999).

[7] C. Nakashima, Z. Yoshida, H. Himura, M. Fukao, J. Morikawa and H. Saitoh, Phys. Rev. E 65, 036409 (2002).
[8] H. Saitoh, Z. Yoshida, C. Nakashima, H. Himura, J. Morikawa and M. Fukao, Phys. Rev. Lett. 92, 255005 (2004).

[9] H. Himura, H. Wakabayashi, Y. Yamamoto et al., Phys. Plasmas 14, 022507 (2007).

[10] J.P. Kremer, T.S. Pedersen, R.G. Lefrancois and Q. Marksteiner, Phys. Rev. Lett. 97, 095003 (2006)

[11] J.P. Kremer, T.S. Pedersen, Q. Marksteiner, R.G. Lefrancois and M. Hahn, Rev. Sci. Instrum. 78, 013503 (2007).

[12] J.P. Marler and M.R. Stoneking, Phys. Rev. Lett. 100, 155001 (2008).

[13] B.N. Ha, M.R. Stoneking and J.P. Marler, Phys. Plasmas 16, 032110 (2009).

[14] S. Pahari, H.S. Ramachandran and P.I. John, Phys. Plasmas 13, 092111 (2006).

[15] M. Amoretti, C. Amsler, G. Bonomi et al., Nature (London) 419, 456 (2002).

[16] G. Gabrielse, N.S. Bowden, P. Oxley et al., Phys. Rev. Lett. 89, 213401 (2002).

[17] N. Kuroda, H.A. Torii, K.Y. Franzen et al., Phys. Rev. Lett. 94, 023401 (2005).

[18] R.G. Greaves and C.M. Surko, Phys. Rev. Lett. 75, 3846 (1995).

[19] J.H. Malmberg and C.F. Driscoll, Phys. Rev. Lett. 44, 654 (1980).

[20] A.J. Theiss, R.A. Mahaffey and A.W. Trivelpiece, Phys. Rev. Lett. 35, 1436 (1975).

[21] Y. Yano, Z. Yoshida, J. Morikawa et al., Plasma Fusion Res. 4, 039 (2009).

[22] H. Saitoh, Z. Yoshida and C. Nakashima, Rev. Sci. Instrum. 73, 87 (2002).

[23] A. Hasegawa, Comments Plasma Phys. Control. Fusion 1, 147 (1987). 